19-098.R1

POULSOM EDITED USA English

ORIGINAL PAPER

\title{
Lineage tracing suggests that ovarian endosalpingiosis does not result from escape of oviductal epithelium
}

\author{
Yisheng Wang ${ }^{1,7} \dagger$, Michael S. Sessine ${ }^{1} \dagger$, Yali Zhai $^{1} \dagger$, Courtney Tipton ${ }^{1}$, Kevin McCool $^{2}$, Rork \\ Kuick $^{3}$, Denise C. Connolly ${ }^{4}$, Eric R. Fearon ${ }^{1,5,6}$, Kathleen R. Cho ${ }^{1,6}$ *
}

Departments of Pathology ${ }^{1}$, Obstetrics and Gynecology ${ }^{2}$, Internal Medicine ${ }^{5}$, and Human Genetics $^{6}$, University of Michigan Medical School, and Department of Biostatistics ${ }^{3}$, University of Michigan School of Public Health, Ann Arbor, MI 48109, Fox Chase Cancer Center ${ }^{4}$, Philadelphia, PA 19111, and Obstetrics \& Gynecology Hospital of Fudan University ${ }^{7}$, Shanghai, PR China, 200090

†Equal contribution

${ }^{*}$ Correspondence to: Kathleen R. Cho, 1504 BSRB, 109 Zina Pitcher Place, University of Michigan, Ann Arbor, MI, 48109-2200 USA. E-mail: kathcho@umich.edu

Running Title: Origins of Ovarian Endosalpingiosis

Conflicts of Interest: The authors declare no conflicts of interest

This is the author manuscript accepted for publication and has undergone full peer review but has not been through the copyediting, typesetting, pagination and proofreading process, which may lead to differences between this version and the Version of Record. Please cite this article as doi: $10.1002 /$ path.5308

This article is protected by copyright. All rights reserved. 


\section{ABSTRACT}

Most high-grade serous carcinomas are thought to arise from Fallopian tube epithelium (FTE), but some likely arise outside of the tube, perhaps from ectopic tubal-type epithelium known as endosalpingiosis. Importantly, the origin of endosalpingiosis is poorly understood. The proximity of the tubal fimbriae to the ovaries has led to the proposal that disruptions in the ovarian surface that occur during ovulation may allow detached FTE to implant in the ovary and form tubal-type glands and cysts. An alternative model suggests that cells present in ectopic locations outside the Müllerian tract retain the capacity for multi-lineage differentiation and can form glands with tubal-type epithelium. We used double transgenic Ovgp1-iCreER ${ }^{T 2} ; R 26 R^{L S L-}$ ${ }^{\text {eYFP }}$ mice, which express an eYFP reporter protein in OVGP1-positive tissues following transient tamoxifen (TAM) treatment, to track the fate of oviductal epithelial cells. Cohorts of adult mice were given TAM to activate eYFP expression in oviductal epithelium, and ovaries were examined at time points ranging from 2 days to 12 months post-TAM. To test whether superovulation might increase acquisition of endosalpingiosis, additional cohorts of TAMtreated mice underwent up to five cycles of superovulation and ovaries were examined at 1 month, 6 months, and 12 months post-TAM. Ovaries were sectioned in their entirety to identify endosalpingiosis. Immunohistochemical staining for PAX8, Tubulin, OVGP1, and eYFP was employed to study endosalpingiosis lesions.

Ovarian endosalpingiosis was identified in $14.2 \%$ of TAM-treated adult mice. The endosalpingiotic inclusion glands and cysts were lined by secretory and ciliated cells and expressed PAX8, tubulin, OVGP1 and eYFP. Neither age nor superovulation was associated with a significant increase in endosalpingiosis. Endosalpingiosis was also occasionally present in the ovaries of pre-pubertal mice. The findings imply that ovarian endosalpingiosis in the mouse does not likely arise as a consequence of detachment and implantation of tubal epithelium and other mechanisms may be relevant.

This article is protected by copyright. All rights reserved. 
Key words: Endosalpingiosis, lineage tracing, Fallopian tube, high-grade serous carcinoma, mouse model

\section{INTRODUCTION}

High-grade serous carcinoma (HGSC), the most common and lethal type of "ovarian" cancer, is now believed to arise most often from Fallopian tube epithelium (FTE), preferentially in the tubal fimbriae [1-5]. However, upwards of one-third of HGSCs lack evidence of FT origin, even after exhaustive microscopic examination of the FTs in women with presumptive ovarian or peritoneal primaries [6]. For example, in their comprehensive review of reports of occult cancers identified in women with hereditary predisposition for ovarian cancer who underwent prophylactic risk-reducing surgery, Yates and colleagues found that $60.5 \%$ were confined to the FTs, whereas $21.1 \%$ involved only the ovary and $2.6 \%$ involved only the peritoneum [7]. These studies suggest that some HGSCs indeed arise outside of the FT mucosa (endosalpinx). Given the absence of data indicating that tubal HGSCs are molecularly or biologically distinct from HGSCs arising in the ovary or peritoneum, it is reasonable to suspect that the latter tumors also arise from FT-like epithelium, albeit in an ectopic location. Endosalpingiosis (ectopic tubal-type epithelium) is a benign entity comprised of the cell types, including ciliated and secretory cells, present in normal FTE [8]. It represents a plausible source of cells that undergo malignant transformation to HGSC in those cases lacking precursor lesions in the tubal fimbriae.

Surprisingly little is known about the origins of endosalpingiosis, and our current understanding is based largely on retrospective analyses of surgical specimens removed for unrelated

This article is protected by copyright. All rights reserved. 
indications. From these observations have emerged two alternative, but not mutually-exclusive models to explain the origin of endosalpingiosis. In the "tubal escape" model,

the close proximity of the tubal fimbriae to the ovarian surface has led to the proposal that disruptions in the ovarian surface epithelium (OSE) that occur during ovulation allow dislodged FTE from the fimbriae to be implanted in the ovary and form inclusion cysts and glands lined by tubal-type epithelium $[8,9]$. Detached FTE might also be exposed to cytokines released during ovulation that attract the cells to the ovary and support their implantation. The "Müllerian metaplasia" model suggests latent cells present in ectopic locations outside the Müllerian tract retain the capacity for multi-lineage differentiation and can reconstitute simple glands with "normal" tubal-type epithelium $[8,10]$.

Importantly, "opportunistic" bilateral salpingectomy and salpingectomy as an alternative to conventional tubal ligation for sterilization have been recommended in Canada and the United States to reduce HGSC risk in women without known hereditary risk [11]. In addition, for women with hereditary ovarian cancer risk, a staged approach (salpingectomy with delayed oophorectomy) for risk reduction is being evaluated $[12,13]$. Each of these cancer prevention strategies relies on the underlying assumption that most HGSCs have a tubal origin. Given existing data supporting the non-tubal origin of at least some HGSCs, it is important to develop a better understanding of the origins of endosalpingiosis and its potential to undergo malignant transformation.

Ovarian endosalpingiosis-like lesions in mouse ovaries have been reported by several groups [14-17]. Genetically engineered mice provide an opportunity to use lineage tracing to monitor the fate of oviductal epithelial cells (equivalent to human FTE) over time, and hence, to determine whether ectopic tubal-type epithelium (e.g., ovarian endosalpingiosis) likely arises as

This article is protected by copyright. All rights reserved. 
a consequence of detachment and implantation in locations outside of the oviducts. The mouse and human anatomy differ in that, in the mouse, the ovaries lie within a membranous pouch (bursa) that isolates them from the peritoneal cavity. The fimbriated end of the oviduct opens into the bursal cavity. Hence, epithelial cells detaching from the distal oviduct cannot easily leave the bursa, have direct access to the ovarian surface, and would be predicted to preferentially implant in the adjacent ovary, particularly if there are breaks in the OSE. OVGP1 is strongly expressed by murine oviductal epithelium, but not by the OSE or cells in the ovarian stroma, and can therefore be considered an excellent marker of tubal differentiation, at least in the mouse [18]. We have recently developed Ovgp1-iCreER ${ }^{T 2}$ mice in which oviduct-specific Cre-mediated recombination in OVGP1-expressing cells can be induced with transient treatment with Tamoxifen (TAM) [18]. We have also generated double transgenic Ovgp1$i C r e E R^{T 2} ; R 26^{L S L-e Y F P}$ mice in which Cre-inducible eYFP reporter expression can be used to monitor the fate of oviductal epithelial cells in TAM-treated mice.

In this study we sought to directly address some key predictions of the proposed models for endosalpingiosis. Specifically, if disruption of the OSE during ovulation allows implantation of detached oviductal epithelium (i.e., "tubal escape") is the dominant mechanism, OVGP1+/eYFP+ endosalpingiosis lesions should increase with age and with increased ovulatory events. In order to test these predictions, we assessed the frequency of endosalpingiosis in the ovaries of TAM-treated double transgenic Ovgp1-iCreER ${ }^{\text {T2 }} ; R_{2} 6^{\text {LSL-eYFP }}$ mice at intervals over 12 months, and in the presence and absence of superovulation.

\section{MATERIALS AND METHODS}

\section{Genetically engineered mice and animal care}


Procedures involving mice for the research described herein have been approved by the University of Michigan's Institutional Animal Care and Use Committee (PRO00006370 and PRO00008343). The development and characterization of Ovgp1-iCreER $R^{T 2}$ transgenic mice and Ovgp1-iCreER ${ }^{T 2}$ transgenic mice crossed with reporter mice carrying the

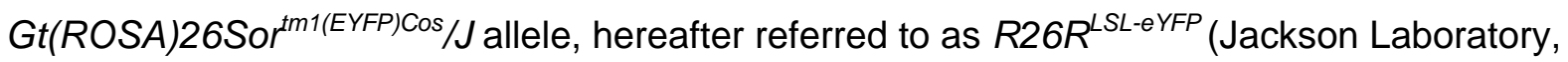
Bar Harbor, ME, USA) have been described in detail [18]. The Ovgp1-iCreER ${ }^{T 2} ; R 26 R^{L S L-e Y F P}$ double transgenic mice were maintained on a mixed genetic background and monitored daily. Mouse genotypes were confirmed by PCR analysis of tail-derived DNA. For all studies, only Ovgp1-iCreER ${ }^{T 2} ; R 26 R^{\text {LSL-eYFP }}$ double transgenic mice were used.

\section{In vivo induction of eYFP expression}

6-8 wk old Ovgp1-iCre-ER ${ }^{T 2} ; R 26 R^{L S L-e Y F P}$ female mice were given intraperitoneal injections of TAM (Sigma-Aldrich, St. Louis, MO) to activate Cre recombinase activity, and hence, eYFP reporter expression in OVGP1 expressing cells/tissues. TAM was first dissolved in 100\% ethanol to a concentration of $400 \mathrm{mg} / \mathrm{ml}$, and then diluted in corn oil to a concentration of 20 $\mathrm{mg} / \mathrm{ml}$, followed by injection at $100 \mathrm{mg} / \mathrm{kg}$ of mouse body weight on each of three consecutive days.

\section{Superovulation}

Cohorts of 6-8 wk old female mice were superovulated as previously described [19]. In brief, mice were given 5 IU pregnant mare serum gonadotropin (PMSG, Sigma-Aldrich) by intraperitoneal injection, followed by 5 IU human chorionic gonadotropin (HCG, Sigma-Aldrich) intraperitoneally $48 \mathrm{~h}$ later. This cycle was initiated 5-7 days following TAM injection and repeated every two weeks for a maximum of 5 cycles. Superovulated mice were sacrificed at intervals $1 \mathrm{mo}, 6 \mathrm{mo}$, or 12 mo post-TAM.

This article is protected by copyright. All rights reserved. 


\section{Histopathology and Immunohistochemistry}

Ovarian and oviductal tissues were fixed in 10\% buffered formalin, paraffin-embedded, and serially sectioned $(5 \mu \mathrm{m})$ in their entirety. Hematoxylin and eosin staining was used to examine the morphology of every third section by routine light microscopy. If any suspected endosalpingiosis lesions were observed, then immunohistochemical (IHC) staining of adjacent sections was used to assess expression of eYFP and OVGP1 in the lesions, with OVGP1 expression confirming tubal-type differentiation in the cells of interest, and eYFP expression indicating their origin from OVGP1 positive cells (presumptively from the FT). Immunohistochemical staining for OVGP1 and PAX8 was also used to study extra-ovarian rete, and in selected cases to clarify the identity of cysts observed near the ovarian hilum. IHC was performed using standard methods as described previously [18]. Primary antibodies used were goat anti-mouse OVGP1 polyclonal antibody (1:500, Santa Cruz Biotechnology, Inc., Santa Cruz, CA, USA), rabbit anti-mouse GFP monoclonal antibody - also detects YFP expression (1:500, Cell Signaling, Danvers, MA, USA), rabbit anti-mouse PAX8 polyclonal antibody (1:750, Novus Biologicals, Englewood, CO, USA), and mouse anti-mouse tubulin monoclonal antibody (1:1000, Sigma-Aldrich). The Mouse on Mouse Detection Kit (Elite Peroxidase, Vector Labs, Burlingame, CA, USA) was used for IHC detection of tubulin following the manufacturer's instructions.

\section{Statistics}

The incidence of ovarian endosalpingiosis lesions in mice $2 \mathrm{~d}$ post-TAM was compared to mice at later time points using the two-tailed Fisher's exact test. Mantel-Haenszel Chi-square and

This article is protected by copyright. All rights reserved. 
Poisson tests for association were used to test for trends in the data on a per ovary or lesion frequency basis, respectively.

\section{RESULTS}

eYFP expression is strongly induced in oviductal epithelium but not OSE following transient TAM treatment of Ovgp1-iCreER ${ }^{T 2} ; R 26 R^{\text {LSL-eYFP }}$ mice

We previously showed, using immunohistochemical (IHC) staining, that OVGP1 is strongly expressed by the oviductal epithelium, but is absent in the ovarian stroma and ovarian surface epithelium and other organs such as liver, lung, pancreas, lymph node and spleen [18]. In our model system, in OVGP1-expressing cells Cre recombinase function can be transiently activated by treating mice with TAM. Cre-mediated recombination removes transcription inhibitory elements, resulting in expression of the eYFP reporter in cells expressing OVGP1. This eYFP expression is retained over the lifetime of the cells and in all of their subsequent progeny. Transient treatment of double transgenic Ovgp1-iCreER ${ }^{T 2} ; R 26 R^{L S L-e Y F P}$ mice with TAM, but not with vehicle, results in strong expression of eYFP in the oviductal epithelium, but not in the ovarian surface epithelium, the underlying ovarian stroma, or adjacent soft tissues (Figure 1A-D and [18]).

\section{Endosalpingiosis-like lesions are present in a subset of ovaries from genetically engineered mice}

In our prior studies aimed at developing various genetically engineered mouse models of ovarian and oviductal carcinomas [18,20-22], we occasionally identified endosalpingiosis-like lesions in mouse ovaries. The lesions which we refer to hereafter as endosalpingiosis are typically characterized by small glands or cysts near the ovarian hilum (Figure 2A), though occasional glands/cysts are present in the subserosal ovarian cortex distant from the hilum

This article is protected by copyright. All rights reserved. 
(Figure 2B). The inclusion cysts/glands are occasionally multiple (Figure 2C) and are lined by OVGP1-positive columnar epithelium including secretory and ciliated cells of the type present in normal oviductal epithelium (Figure 2D). The epithelium lining the glands/cysts is focally positive for tubulin, which marks ciliated cells (Figure 2E), and PAX8 which preferentially marks secretory cells (Figure 2F).

\section{Mouse ovarian endosalpingiosis lesions do not represent rete ovarii}

As noted above, we observed that most ovarian endosalpingiosis lesions in adult mice (with or without superovulation) were located near the hilum or had at least one side of the cyst wall facing the hilum when the cyst was too large to determine its location with certainty. Their predominantly hilar location suggests the possibility that the lesions represent mesonephric duct remnants (rete anlage), which have been proposed as an origin of cysts and proliferative epithelial lesions in the mouse ovary and can occasionally show cilia [23]. We wished to determine whether the mouse endosalpingiosis lesions in ovaries might represent dilated rete ovarii. An example of extra-ovarian rete in periadnexal soft tissue adjacent to the oviduct and ovary is shown in Figure 3. We found the extra-ovarian rete (Figure $3 A, B$ ) is PAX8 positive (Figure 3C, D) and OVGP1 negative (Figure 3E, F), while all of the endosalpingiosis lesions were OVGP1 positive. These findings indicate the ovarian endosalpingiosis lesions in the mice do not likely represent dilated rete ovarii.

\section{Ovarian endosalpingiosis in Ovgp1-iCreER ${ }^{T 2} ; R 26 R^{\text {LSL-eYFP }}$ mice does not increase with age}

We initially evaluated a cohort of mice ( $n=18,36$ ovaries) only 2 days after the sequential 3-day treatment of mice with TAM. By light microscopic analysis, endosalpingiosis lesions were found in $11.1 \%$ (2 of 18) animals; an example is shown in Figure 4A,B. As expected, the

This article is protected by copyright. All rights reserved. 
lesions expressed OVGP1, based on IHC staining (Figure 4C,D). Interestingly, cells in the ovarian endosalpingiosis lesions were also eYFP positive (Figure 4E,F); eYFP positive cells from the oviduct might have detached and subsequently implanted in the ovary within only 2 days of TAM treatment (tubal escape). However, based on the number of cells in the lesions and the very short time post-TAM, it is much more likely that the OVGP1 positive cells were already resident in the ovary at the time of TAM treatment (Müllerian metaplasia). If "tubal escape" were the dominant mechanism underlying mouse ovarian endosalpingiosis, we had predicted that eYFP positive lesions would accumulate in double transgenic Ovgp1$i C r e E R^{T 2} ; R 26 R^{L S L-e Y F P}$ mice as they aged. Cohorts of Ovgp1-iCreER ${ }^{T 2} ; R 26 R^{L S L-e Y F P}$ mice were given TAM injections at $6-8$ weeks of age and then sacrificed $1 \mathrm{wk}(n=12), 1 \mathrm{mo}(\mathrm{n}=9), 6 \mathrm{mo}$ $(n=20)$ and 12 mo $(n=20)$ after transient TAM treatment. Both ovaries and oviducts from each animal were harvested and sectioned in their entirety as described above. OVGP1/eYFP double-positive endosalpingiosis lesions were identified in $8.3 \%, 11.1 \%, 10 \%$ and $20 \%$ of animals in these cohorts, respectively (Table 1). None of the mice had endosalpingiosis in both ovaries and the number of lesions present in a given ovary was two or fewer. Although there was a trend toward increased endosalpingiosis (e.g., between $1 \mathrm{wk}$ and 12 mo post-TAM), this was not statistically significant ( $P=0.51$ Mantel-Haenszel Chi-square test of association), and no time points were significantly different from the results for day 2 mice (Table 1, two-sided Fisher Exact tests all gave $P>0.6$ ). No OVGP1 positive/eYFP negative or eYFP positive/OVGP1 negative lesions were identified.

\section{Ovarian endosalpingiosis in Ovgp1-iCreER ${ }^{T 2} ; R 26 R^{\text {LSL-eYFP }}$ mice does not increase with superovulation}

This article is protected by copyright. All rights reserved. 
The "tubal escape" hypothesis also predicts that superovulated mice will harbor more ovarian endosalpingiosis than control mice, and that these lesions will accumulate over time. To test this experimentally, littermates of mice described above were used to generate additional cohorts of mice that underwent up to 5 cycles of superovulation and these cohorts were sacrificed at $1 \mathrm{mo}(\mathrm{n}=8), 6 \mathrm{mo}(\mathrm{n}=22)$ and $12 \mathrm{mon}(\mathrm{n}=18)$ post-TAM. Endosalpingiosis lesions were identified in $25 \%, 18.2 \%$, and $11.1 \%$ of animals in these cohorts, respectively, indicating that endosalpingiosis does not accumulate in superovulated mice and providing further evidence that mouse endosalpingiosis does not accumulate with age (Table 1, $P=1.0$ for the 4 by 2 table that included day 2 control mice, Mantel-Haenszel Chi-square test of association), nor did mice undergoing superovulation have endosalpingiosis more frequently than control mice at the same time points ( $P>0.5$ for all three time points, two-sided Fisher Exact test). All of the lesions identified were OVGP1/eYFP double-positive. One mouse had endosalpingiosis in both ovaries and the maximum number of lesions in a given ovary was three (seen in one mouse). Overall, ovarian endosalpingiosis was identified in 14.2\% (18 of 127) of the adult mice included in our study (Table 1).

\section{Mouse ovarian endosalpingiosis is observed before sexual maturation}

In order to determine whether mouse ovarian endosalpingiosis lesions can occur prior to sexual maturity (i.e., requires ovulation), and whether OVGP1-positive cells are already present in the ovaries of prepubertal mice, a cohort $(n=30)$ of 4 wk old mice was treated with TAM and sacrificed 3-4 days later. This time point precedes sexual maturation [24]. In this cohort, ovarian endosalpingiosis was identified in 2 of 30 animals (6.7\%). Comparison of endosalpingiosis frequency in this group to the day 2 mice gives $P=0.62$ (two-sided Fisher Exact test). These lesions were also OVGP1/eYFP double positive, and were located in the

This article is protected by copyright. All rights reserved. 
ovarian medulla near the hilum. These results suggest that mouse ovarian endosalpingiosis is present early in post-natal development, does not require ovulation, and that OVGP1 expression does not require the presence of adult levels of ovarian hormones.

\section{DISCUSSION}

Our studies show that mice acquire ovarian endosalpingiosis-like lesions, and that these lesions do not likely arise as a consequence of detachment from the oviduct followed by implantation in the ovary. If an implantation mechanism for endosalpingiosis is not supported by our data, what other mechanisms should be considered? Presumably, ectopic tubal-type epithelium could arise from differentiation of resident pluripotent or multipotent stem-like cells, or as a consequence of metaplasia (trans-differentiation), in which one type of differentiated cell changes to another cell type [25]. In either case, this differentiation "switch" would likely depend on the appropriate local and/or systemic hormonal milieu, as endosalpingiosis is uncommon in human males $[26,27]$. However, our identification of ovarian endosalpingiosis in a subset of pre-pubertal mice suggests that development of endosalpingiosis is not dependent on post-pubertal hormones, at least in mice. The propensity for endosalpingiosis-like lesions in mice to arise near the ovarian hilum could reflect tubal-type differentiation of resident cells with stem-like properties that are enriched in this region [28]. Specifically, Flesken-Nikitin and colleagues found that the hilar/junctional area between the OSE, mesothelium and tubal (oviductal) epithelium is enriched for cells that express stem and/or progenitor cell markers such as ALDH1, LGR5, LEF1, CD133 and CK6B, and display stem cell properties ex vivo and in vivo. Hence, under the right conditions, these cells could potentially acquire tubal-type differentiation manifest as glands and cysts that initiate in the ovarian hilum. Presumably, this

This article is protected by copyright. All rights reserved. 
tubal type epithelium was already present in the ovaries in a subset of mice, before activation of the eYFP reporter in OVGP1-positive cells induced by TAM.

Importantly, mice and humans have anatomical and biological differences such that conclusions drawn in one mammalian system may not apply to the other and we cannot exclude detachment and implantation of FTE as an important underlying mechanism of endosalpingiosis in humans. However, the occasional identification of endosalpingiosis in human males [29], argues against "tubal escape" as the sole mechanism underlying endosalpingiosis in women. Interestingly, while ovarian endosalpingiosis in the mouse might be expected to arise from either implantation or Müllerian metaplasia, endosalpingiosis outside the ovary, e.g., the omentum, presumably occurs only via the latter mechanism, since detached FTE from the oviduct cannot readily access the peritoneal space in mice due to the presence of the murine ovarian bursa. This contrasts with the situation in humans, in which detached FTE has direct access to the peritoneal cavity. We have occasionally observed endosalpingiosislike lesions outside of the ovary in mice (Supplemental Figure 1), but this has been in the context of oviductal tumor development in Ovgp1-iCreER ${ }^{T 2}$ mice also carrying tumor suppressor genes alleles that undergo recombination following TAM treatment. Though this finding would provide further support for stem cell differentiation or metaplasia rather than tubal escape as the primary mechanism underlying endosalpingiosis in mice, more in-depth analysis of endosalpingiosis-like lesions in extra-ovarian locations are needed before meaningful conclusions can be drawn.

Our data fail to support detachment and implantation of oviductal epithelium as a major mechanism underlying endosalpingiosis in mice. In 2005, Bristol-Gould and colleagues described a mouse model of ovarian endosalpingiosis in which the Müllerian-inhibiting 
substance (MIS) promoter was used to drive expression of a dominant-negative form of SMAD2 [16]. In this model system, female mice develop ovarian endosalpingiosis and the authors proposed that the lesions likely arise from Müllerian epithelium, though the origin of the lesional cells (e.g., oviduct, endometrium, rete ovarii, or OSE) was not determined. More recently, Gendronneau et al reported ovarian "inclusion cysts reminiscent of human endosalpingiosis" in Hoxa5 ${ }^{--}$female mice [17]. The epithelium lining the cysts was shown to express WT1 and PAX8, consistent with Müllerian tubal-type differentiation. Other studies have described analyses of serous epithelial inclusion cysts in mouse ovaries in relation to age, pregnancy, and/or number of ovulations $[14,15]$. Interestingly, Burdette and colleagues studied ovarian "inclusion" cysts with features resembling endosalpingiosis in chronically superovulated wild-type CD1 mice and CD1 mice carrying a dominant negative Smad2 transgene under control of the MISIIR promoter. They found that the number of ovarian "inclusion cysts" was not significantly different between superovulated and control mice in either the wild-type or transgenic groups [15]. While they demonstrated that the observed cysts were epithelial (CK8 positive), they were not able to identify the origin of the cysts due to lack of specific markers. These prior studies by Gendronneau and Burdette utilized transgenic animals with genetically engineered alleles of powerful modulators of cell fate and differentiation. An important strength of the model described here is the use of lineage tracing with an eYFP reporter that is not predicted to alter cell identity or differentiation capacity. However, we cannot completely exclude the possibility that eYFP itself has toxic effects in some microenvironmental contexts, and this may have limited our ability to observe an accumulation of $e Y F P^{+}$lesions in ovarian locations. Importantly, others have shown that YFP, but not GFP, provides stable fluorescent marking of rodent hepatic adult stem cells [30]. It also bears noting that we observed eYFP ${ }^{+}$ lesions up to 12 months after TAM treatment, suggesting that the presence of intracellular eYFP is not inherently toxic to cells.

This article is protected by copyright. All rights reserved. 
Finally, Tan et al reported that cysts resembling benign serous inclusion cysts in humans increase with age and with incessant ovulation in out-bred Swiss Webster mice, but proposed the OSE rather than the oviductal epithelium as the likely origin of the cysts [14]. Additional approaches and experimental work will be needed to address the relative contribution of the OSE to endosalpingiosis-like lesions in the mouse. Our work confirms earlier findings in mice and provides data suggesting that the "inclusion" glands and cysts in the mouse ovary with features resembling endosalpingiosis do not arise as a consequence of oviductal epithelial detachment and subsequent implantation in the ovary.

\section{ACKNOWLEDGEMENTS}

Research reported in this publication was supported by the National Cancer Institute of the National Institutes of Health under Award Numbers P30CA046592 (KRC, ERF, RK) including use of the Transgenic Animal Models and Biostatistics, Analytics \& Bioinformatics Shared Resources, and R01CA188516 (KRC, YZ, RK).

\section{AUTHOR CONTRIBUTIONS STATEMENT}

YW, MS, YZ, CT, KM, RK, DCC, ERF, and KRC conceived experiments and/or analyzed data. YW, MS, CT, and YZ conceived and carried out experiments. All authors were involved in writing the paper and had final approval of the submitted version.

This article is protected by copyright. All rights reserved. 


\section{REFERENCES}

1. Piek JM, van Diest PJ, Zweemer RP, et al. Dysplastic changes in prophylactically removed Fallopian tubes of women predisposed to developing ovarian cancer. J Pathol 2001; 195: 451456.

2. Crum CP, Drapkin R, Miron A, et al. The distal fallopian tube: a new model for pelvic serous carcinogenesis. Curr Opin Obstet Gynecol 2007; 19: 3-9.

3. Kindelberger DW, Lee Y, Miron A, et al. Intraepithelial carcinoma of the fimbria and pelvic serous carcinoma: Evidence for a causal relationship. Am J Surg Pathol 2007; 31: 161-169.

4. Lee $\mathrm{Y}$, Miron A, Drapkin R, et al. A candidate precursor to serous carcinoma that originates in the distal fallopian tube. J Pathol 2007; 211: 26-35.

5. Medeiros F, Muto MG, Lee $\mathrm{Y}$, et al. The tubal fimbria is a preferred site for early adenocarcinoma in women with familial ovarian cancer syndrome. Am J Surg Pathol 2006; 30: $230-236$.

6. Przybycin CG, Kurman RJ, Ronnett BM, et al. Are all pelvic (nonuterine) serous carcinomas of tubal origin? Am J Surg Pathol 2010; 34: 1407-1416.

7. Yates MS, Meyer LA, Deavers MT, et al. Microscopic and early-stage ovarian cancers in BRCA1/2 mutation carriers: building a model for early BRCA-associated tumorigenesis. Cancer prevention research 2011; 4: 463-470.

8. Prentice L, Stewart A, Mohiuddin S, et al. What is endosalpingiosis? Fertil Steril 2012; 98: 942947.

9. Vang R, Shih I-M, Kurman RJ. Fallopian tube precursors of ovarian low- and high-grade serous neoplasms. Histopathology 2013; 62: 44-58.

This article is protected by copyright. All rights reserved. 
10. Kurman RJ, Shih le M. The origin and pathogenesis of epithelial ovarian cancer: a proposed unifying theory. Am J Surg Pathol 2010; 34: 433-443.

11. McAlpine JN, Hanley GE, Woo MM, et al. Opportunistic salpingectomy: uptake, risks, and complications of a regional initiative for ovarian cancer prevention. Am J Obstet Gynecol 2014; 210: 471 e471-411.

12. Holman LL, Friedman S, Daniels MS, et al. Acceptability of prophylactic salpingectomy with delayed oophorectomy as risk-reducing surgery among BRCA mutation carriers. Gynecol Oncol 2014; 133: 283-286.

13. Nebgen DR, Hurteau J, Holman LL, et al. Bilateral salpingectomy with delayed oophorectomy for ovarian cancer risk reduction: A pilot study in women with BRCA1/2 mutations. Gynecol Oncol 2018; 150: 79-84.

14. Tan OL, Hurst PR, Fleming JS. Location of inclusion cysts in mouse ovaries in relation to age, pregnancy, and total ovulation number: implications for ovarian cancer? J Pathol 2005; 205: 483-490.

15. Burdette JE, Oliver RM, Ulyanov V, et al. Ovarian epithelial inclusion cysts in chronically superovulated CD1 and Smad2 dominant-negative mice. Endocrinology 2007; 148: 3595-3604.

16. Bristol-Gould SK, Hutten CG, Sturgis C, et al. The development of a mouse model of ovarian endosalpingiosis. Endocrinology 2005; 146: 5228-5236.

17. Gendronneau G, Boucherat O, Aubin J, et al. The loss of Hoxa5 function causes estrous acyclicity and ovarian epithelial inclusion cysts. Endocrinology 2012; 153: 1484-1497.

18. Wu R, Zhai Y, Kuick R, et al. Impact of oviductal versus ovarian epithelial cell of origin on ovarian endometrioid carcinoma phenotype in the mouse. J Pathol 2016; 240: 341-351.

This article is protected by copyright. All rights reserved. 
19. Choi KH, Joo BS, Sun ST, et al. Administration of visfatin during superovulation improves developmental competency of oocytes and fertility potential in aged female mice. Fertil Steril 2012; 97: 1234-1241 e1231-1233.

20. Zhai $Y$, Wu R, Kuick R, et al. High-grade serous carcinomas arise in the mouse oviduct via defects linked to the human disease. J Pathol 2017; 243: 16-25.

21. Wu R, Hendrix-Lucas N, Kuick R, et al. Mouse model of human ovarian endometrioid adenocarcinoma based on somatic defects in the Wnt/B-catanin and PI3K/Pten signaling pathways. Cancer Cell 2007; 11: 321-333.

22. Zhai $Y$, Kuick R, Tipton $C$, et al. Arid1a inactivation in an Apc- and Pten-defective mouse ovarian cancer model enhances epithelial differentiation and prolongs survival. J Pathol 2015; 238: 2130.

23. Long GG. Apparent mesonephric duct (rete anlage) origin for cysts and proliferative epithelial lesions in the mouse ovary. Toxicol Pathol 2002; 30: 592-598.

24. Dutta S, Sengupta P. Men and mice: Relating their ages. Life Sci 2016; 152: 244-248.

25. Karnezis AN, Cho KR, Gilks CB, et al. The disparate origins of ovarian cancers: pathogenesis and prevention strategies. Nature Rev Cancer 2016; 17: 65-74.

26. Zinsser KR, Wheeler JE. Endosalpingiosis in the omentum: a study of autopsy and surgical material. Am J Surg Pathol 1982; 6: 109-117.

27. Irving J, Clement PB. Diseases of the Peritoneum. In: Blaustein's Pathology of the Female Genital Tract. 6th ed. Kurman RJ, Ellenson LH, Ronnett B, (eds). Springer: New York, 2011.

28. Flesken-Nikitin $\mathrm{A}$, Hwang $\mathrm{Cl}$, Cheng $\mathrm{CY}$, et al. Ovarian surface epithelium at the junction area contains a cancer-prone stem cell niche. Nature 2013; 495: 241-245.

This article is protected by copyright. All rights reserved. 
29. Gallan AJ, Antic T. Benign mullerian glandular inclusions in men undergoing pelvic lymph node dissection. Hum Pathol 2016; 57: 136-139.

30. Taghizadeh RR, Sherley JL. CFP and YFP, but not GFP, provide stable fluorescent marking of rat hepatic adult stem cells. J Biomedicine Biotechnol 2008; 2008: 453590.

This article is protected by copyright. All rights reserved. 


\section{FIGURE LEGENDS}

Figure 1. Tamoxifen induces eYFP expression in the oviductal epithelium of Ovgp1iCreER $R^{T 2} ; R 26 R^{L S L-e Y F P}$ mice. Oviductal expression of eYFP (seen as green fluorescence) is induced by transient treatment with TAM (A) but not vehicle (B) in Ovgp1-iCreE $R^{T 2} ; R 26 R^{L S L-e Y F P}$ double transgenic mice. Mouse oviducts are indicated by white arrows, ovaries by white arrow heads and uterine horns by white stars. Representative photomicrographs showing eYFP expression in the oviductal epithelium of TAM-treated mouse based on immunohistochemical staining (C), but not in the vehicle treated mouse (D).

Figure 2. Representative examples of ovarian endosalpingiosis in Ovgp1$i C r e E R^{T 2} ; R 26 R^{L S L-e Y F P}$ mice. Ovarian endosalpingiosis lesions (indicated by black stars), are usually located in the medulla near ovarian hilum (A), but are occasionally found in the ovarian cortex (B). Some ovaries harbor more than one lesion (C). The epithelium lining endosalpingiotic glands and cysts is OVGP1 positive (inset) and includes both secretory and ciliated cells (D), with ciliated cells expressing tubulin (E) and secretory cells preferentially expressing PAX8 (F, inset shows high-magnification). Note the internal positive control for PAX8 (normal oviduct) in lower left corner of panel F at black arrow. Black arrowheads indicate ciliated cells in panels D and E. Scale bars: $200 \mu \mathrm{m}$ (panels A, B, F); $100 \mu \mathrm{m}$ (panel C); $50 \mu \mathrm{m}$ (panels D, E).

Figure 3. Endosalpingiosis lesions in Ovgp1-iCreER ${ }^{T 2} ; R 26 R^{L S L-e Y F P}$ mice are different from rete ovarii. Although extra-ovarian rete also forms glands lined by columnar cells $(A)$ that express PAX8 (C), the rete does not express OVGP1 (E). High magnifications of boxed areas

This article is protected by copyright. All rights reserved. 
in $A, C$, and $E$ are shown in $B, D, F$, respectively. Note the internal positive control for detection of PAX8 and OVGP1 expression (normal oviduct at black arrows) in lower left corners of panels C and E. Scale bars: $200 \mu \mathrm{m}$ (panels A, C, E); $50 \mu \mathrm{m}$ (panels B, D, F).

Figure 4. Ovarian endosalpingiosis lesions express both OVGP1 and eYFP in TAMtreated Ovgp1-iCreER ${ }^{T 2} ; R 26 R^{L S L-e Y F P}$ mice. A representative example of endosalpingiosis in a TAM-treated double transgenic mouse is shown: H\&E stained section (A), and adjacent sections immunohistochemically stained for OVGP1 (C) and eYFP (E). High magnifications of boxed areas in panels $A, C$, and $E$ are shown in panels $B, D$, and F, respectively. Note the internal positive control for detection of OVGP1 and eYFP expression (normal oviduct at black arrows) in lower left corners of panels C and E. Scale bars: $200 \mu \mathrm{m}$ (panels A, C, E); $50 \mu \mathrm{m}$ (panels B, D, F).

SUPPLEMENTARY MATERIAL ONLINE

Supplementary materials and methods NO

Supplementary figure legends NO, the legend is embedded

Figure S1. Endosalpingiosis-like lesions are occasionally observed in the omentum

This article is protected by copyright. All rights reserved. 


\begin{tabular}{|c|c|c|c|c|c|c|c|}
\hline \multirow[b]{2}{*}{ Time post-TAM } & \multicolumn{3}{|c|}{ No superovulation } & \multicolumn{3}{|c|}{ Superovulation } & \multirow[b]{2}{*}{$\begin{array}{c}P \text {-value } \\
\text { versus } \\
\text { controls } \\
\text { at the } \\
\text { same } \\
\text { time* }\end{array}$} \\
\hline & $\begin{array}{l}\text { \# of mice } \\
\text { with } \\
\text { lesion / } \\
\text { total } \\
\text { number of } \\
\text { mice }\end{array}$ & $\begin{array}{c}\% \\
\text { with } \\
\text { lesion }\end{array}$ & $\begin{array}{c}P \text {-value } \\
\text { versus } 2 \\
\text { day post- } \\
\text { TAM } \\
\text { controls* }\end{array}$ & $\begin{array}{l}\text { \# of mice } \\
\text { with } \\
\text { lesion / } \\
\text { total } \\
\text { number of } \\
\text { mice }\end{array}$ & $\begin{array}{c}\% \\
\text { with } \\
\text { lesion }\end{array}$ & $\begin{array}{c}P \text {-value } \\
\text { versus } 2 \\
\text { day post- } \\
\text { TAM } \\
\text { controls* }\end{array}$ & \\
\hline 2 days & $2 / 18$ & $11.1 \%$ & & & & & \\
\hline 1 week & $1 / 12$ & $8.3 \%$ & 1.00 & & & & \\
\hline 1 month & $1 / 9$ & $11.1 \%$ & 1.00 & $2 / 8$ & $25.0 \%$ & 0.56 & 0.58 \\
\hline 6 months & $2 / 20$ & $10.0 \%$ & 1.00 & $4 / 22$ & $18.2 \%$ & 0.67 & 0.67 \\
\hline 12 months & $4 / 20$ & $20.0 \%$ & 0.66 & $2 / 18$ & $11.1 \%$ & 1.00 & 0.66 \\
\hline
\end{tabular}

Table 1. Incidence of endosalpingiosis in mice over time, with and without superovulation

*All $P$-values are from two-sided Fisher Exact Tests

This article is protected by copyright. All rights reserved. 

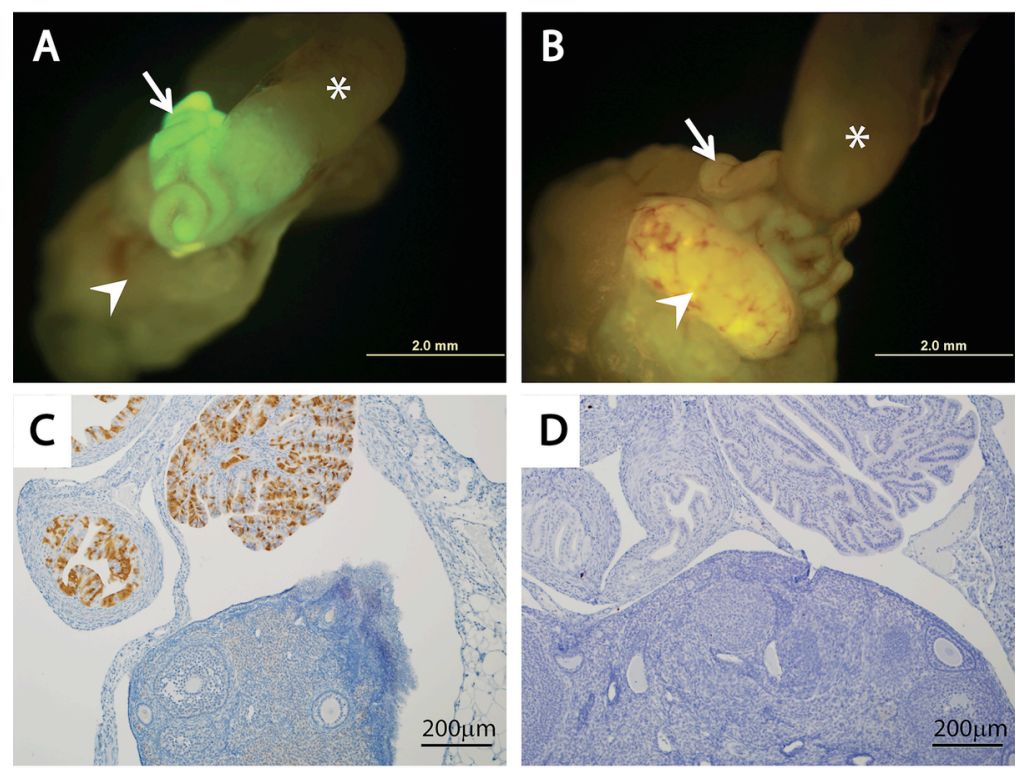

D

PATH_5308_Figure 1-s.tif

This article is protected by copyright. All rights reserved. 

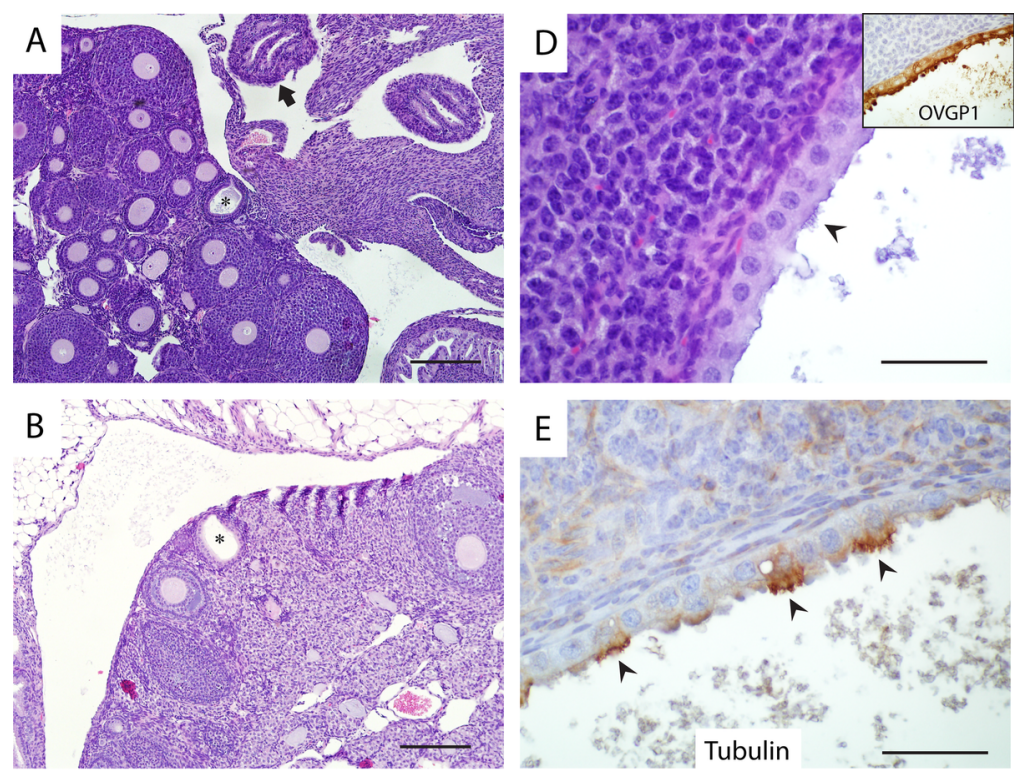

E
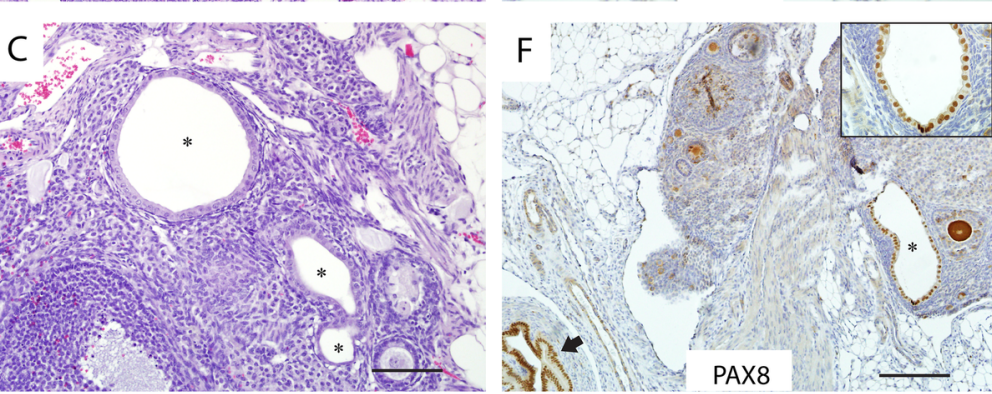

PATH_5308_Figure 2-s.tif

This article is protected by copyright. All rights reserved. 

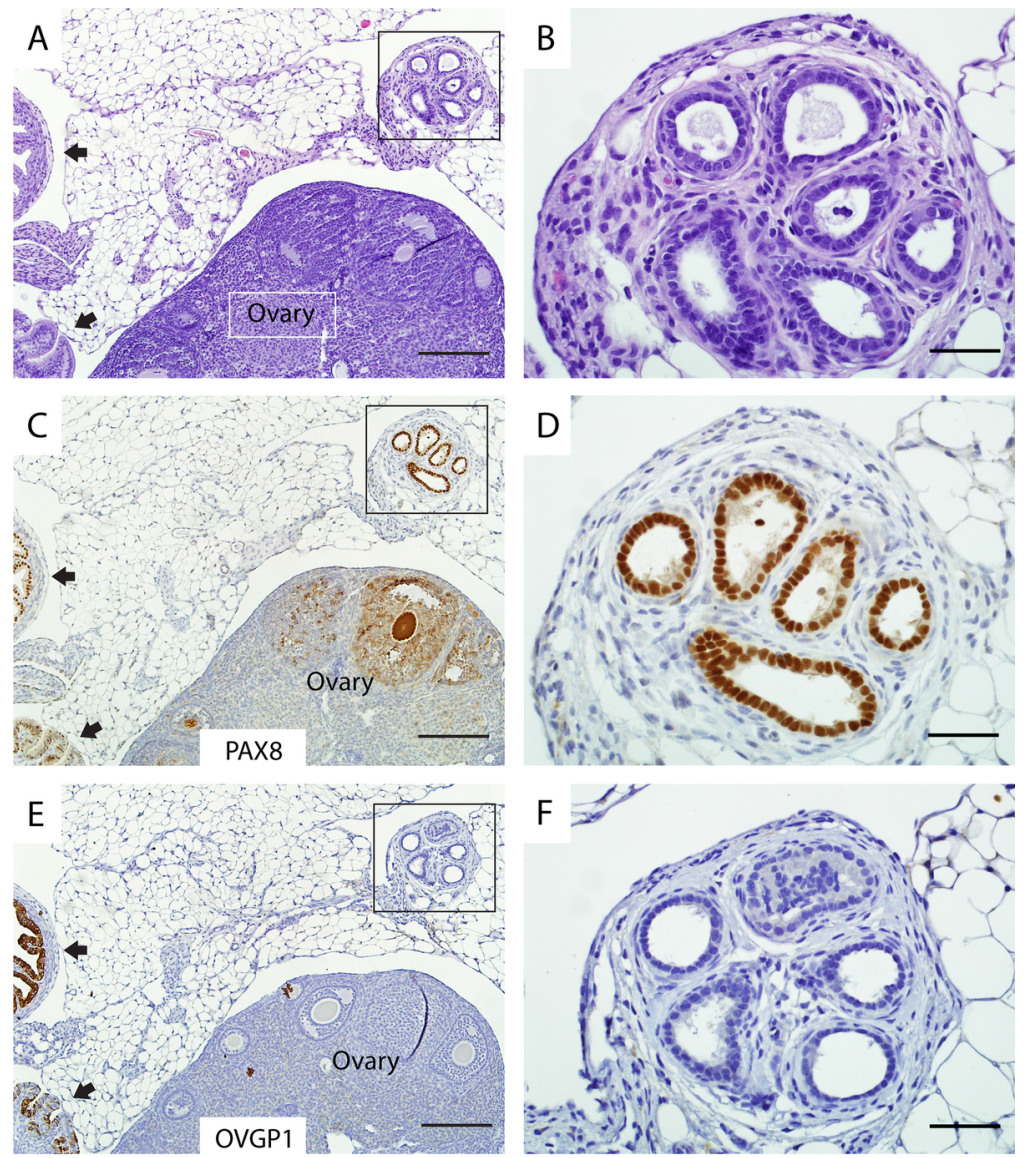

PATH_5308_Figure 3-s.tif

This article is protected by copyright. All rights reserved. 

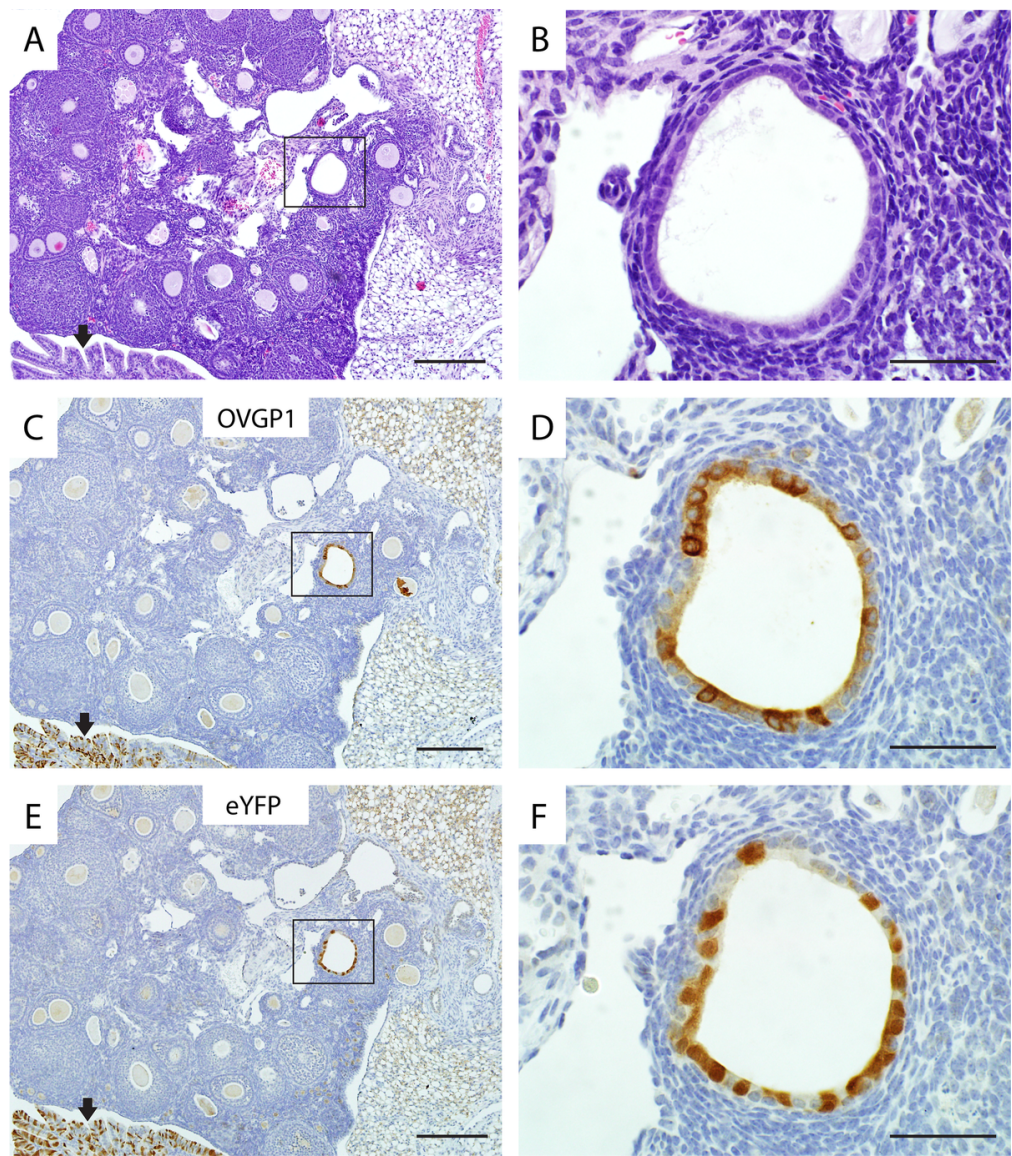

PATH_5308_Figure 4-s.tif

This article is protected by copyright. All rights reserved. 